\title{
LA EXPANSIÓN DEL AFIJO VELAR /G/ EN CATALÁN ENTRE LOS SIGLOS XIII Y XV*
}

\author{
Manuel Badal \\ Universitat de València
}

\section{RESUMEN}

En este trabajo, analizamos la expansión del afijo velar /g/ en catalán, que pasa a ser la marca de una subclase de verbos de la segunda conjugación. La velar procedía inicialmente o bien de la consonante final del tema de presente: DICO > dic 'digo', o bien de la consonantización de la marca aspectual de los verbos con perfecto fuerte en -UI-: DĒBUIT > dec >> degué 'debió'. La propagación del afijo se produce desde las formas del tema de presente hasta las formas del tema de perfecto y viceversa. Para justificar esta extensión, nos basamos en el concepto de exaptación (Lass 1990), que se define como la reutilización de material morfológico no funcional, que pasa a desarrollar una nueva función no prevista en la gramática. La exaptación se produjo cuando la velar asociada al tema de perfecto dejó de funcionar como afijo aspectual y se unificó con la velar que aparecía en la raíz de algunos verbos en el tema de presente, convirtiéndose en una marca de subclase verbal. Para apoyar esta tesis, aportamos documentación de dicha extensión en tres verbos que representan tres subclases diferentes: verbos en que la velar aparece inicialmente en el tema de presente (dir); verbos en que aparece en las formas de perfecto (deure) y verbos en que no aparece originariamente en ninguna forma (prendre).

Palabras Clave: diacronía, exaptación, morfología verbal, velarización, catalán.

\author{
THE VELAR AFFIX /G/ EXPANSION IN CATALAN \\ BETWEEN THE $13^{\text {TH }}$ AND $15^{\text {TH }}$ CENTURIES
}

\section{Abstract}

In this paper, we analyze the expansion of the velar affix /g/ in Catalan, which becomes the mark of a verbal subclass of the second conjugation. The velar originated either from the final consonant of the present theme: DICO > dic 'I say', or from the consonantization of the aspectual mark of verbs with a strong perfect in -UI-: DĒBUIT > dec >> degué 'ought'. The affix propagation occurs both from the present theme forms to the perfect theme forms and vice versa. To justify this change, we employ the concept of exaptation (Lass 1990), which is defined as the reuse of non-functional morphological material that ends up developing a new function not foreseen by the grammar. The exaptation occurred when the perfect theme velar stopped functioning as an aspectual affix, was unified with the velar from the present theme and became a verbal subclass mark. To support this thesis, we provide documentation of the velar extension in three verbs representing three different subclasses: verbs with velar in the present theme (dir); verbs with velar in the perfective forms (deure), and verbs in which it does not appear originally in any form (prendre).

KEYwORDs: diachrony, exaptation, verbal morphology, velarization, Catalan.

DOI: https://doi.org/10.25145/j.refiull.2020.40.03 


\section{INTRODUCCIÓN}

Dentro de la segunda conjugación del catalán encontramos una subclase verbal que se caracteriza por una gran sistematicidad y estabilidad: se trata de la clase de verbos velarizados como beure 'beber', deure 'deber' o dir 'decir'. Muchos autores han dedicado tiempo y trabajo a estudiarla, como Mascaró (1983, 1985), Pérez Saldanya (1996, 1998, 2013), Querol (2011), Viaplana (1986) o Wheeler (2011). En este apartado introductorio presentamos la extensión del afijo velar en el catalán actual y su origen.

En el catalán normativo actual, el afijo velar $(/ \mathrm{g} /)$ se presenta en las personas que indicamos en la tabla 1, en la que ilustramos con el verbo deure 'deber' las formas en que aparece. En los tiempos en que todas las formas están velarizadas (presente de subjuntivo, pretérito perfecto simple e imperfecto de subjuntivo) solo ejemplificamos la tercera persona. Dentro de esta subclase verbal, el único caso en que suele haber vacilación entre formas velarizadas y no velarizadas es el participio. De esta manera, mientras que el participio de deure está velarizado, los otros dos verbos que vamos a utilizar como referencia a lo largo del artículo, dir 'decir' y prendre 'tomar', aún conservan un participio fuerte sin velarizar: dit 'dicho' y pres 'tomado', respectivamente'.

\begin{tabular}{|c|c|c|}
\hline \multicolumn{3}{|c|}{$\begin{array}{l}\text { TABLA 1. FORMAS VELARIZADAS DEL VERBO DEURE 'DEBER', COMPARADAS } \\
\text { CON LAS FORMAS EQUIVALENTES DEL VERBO NO VELARIZADO BATRE 'BATIR' }\end{array}$} \\
\hline Tiempo Verbal & FORMA VELARIZADA & \\
\hline 1. ${ }^{\mathrm{a}}$ persona del presente de indicativo & 1 dec- $\varnothing$ & 1 bat $\varnothing$ \\
\hline Presente de subjuntivo & 3 deg-a & $3 b a t-a$ \\
\hline 3. ${ }^{\mathrm{a}}, 4 .^{\mathrm{a}}$ y $6{ }^{\mathrm{a}}$ personas del imperativo* & 3 deg-a, 4 degu-em, 6 degu-en & 3 bat-a, 4 bat-em, 6 bat-en \\
\hline Pretérito perfecto simple & 3 degu-é & 3 bat-é \\
\hline
\end{tabular}

* Este estudio, financiado por el programa de subvenciones para la contratación de personal investigador de carácter predoctoral (ACIF/2017) de la Generalitat Valenciana, forma parte del proyecto FFI2016-76245-C3-3-P, financiado por la Agencia Estatal de Investigación y por el FEDER (http://www.ub.edu/GEVAD), y del grupo GIUV2017-397, de la Universitat de València. Agradezco los comentarios de los asistentes al XV Encuentro de Morfólogos Españoles (Universidad de La Laguna, 9-10/05/2019), así como las sugerencias de dos revisores anónimos y de Jesús Jiménez, Maria-Rosa Lloret y Manuel Pérez Saldanya.

${ }_{1}^{1}$ En los verbos del modelo de deure, a partir de antiguos perfectos velarizados como dec 'debió', se produjo un proceso de expansión hacia los participios débiles de esta subclase, originalmente sin velarizar: DĒBITU $>$ *DĒBŪTŪ $>$ deüt $>$ degut 'debido'. Esta extensión reforzaba la relación entre el participio y el pretérito perfecto simple (ya velarizado: $d e c$ ), evitaba el hiato en el participio (deüt [de.út]) y, por lo tanto, otorgaba una estructura silábica menos marcada al participio (Pérez Saldanya 1998: 251). A pesar de todas estas ventajas, en un grupo numeroso de verbos se han mantenido los participios fuertes sin velarizar, como en dit y pres; es el caso, p. ej., de los participios que en latín presentaban una terminación en -sU, como INCENSU > encés 'encendido', o los que contienen el grupo -CT-, como DUCTU >> dut 'llevado'. 


\begin{tabular}{|c|c|c|}
\hline Imperfecto de subjuntivo & 3 degu-és/degu-era & 3 bat-és/bat-era \\
\hline Participio & deg-ut & bat-ut \\
\hline
\end{tabular}

En cuanto al origen de la consonante velar, el catalán presenta formas con este afijo que provienen o bien del tema de presente latino, esto es, presente de indicativo y presente de subjuntivo, o bien del tema de perfecto, es decir, pretérito perfecto simple y pluscuamperfectos de indicativo y de subjuntivo latinos. Estos dos últimos tiempos se convertirán, más adelante, en el condicional y el imperfecto de subjuntivo del catalán antiguo. En el tema de presente, en los verbos en los que la velar es etimológica, esta corresponde al segmento final del radical (Dīc-ō). Dentro de este sistema, encontramos tres grupos: el primero, formado por aquellos verbos en que la velar del étimo latino estaba precedida de una vocal: p. ej., Dīcō > dic 'digo'; el segundo, formado por los verbos en que la velar iba precedida de una consonante nasal p. ej., PLANGō > planc 'plaño', y, el tercero, formado por los llamados incoativos de radical: p. ej., CRĒSCO > cresc 'crezco'. En las tablas 2, 3 y 4 podemos ver los paradigmas del tema de presente del catalán antiguo. En los tres grupos, por razones fonéticas, la consonante velar se mantenía en catalán medieval en la primera persona del presente de indicativo y en el presente de subjuntivo. Hemos sombreado las celdas de las personas en que el afijo velar aparece. A esta distribución se le ha dado el nombre de patrón L (Maiden 2018: 84), ya que las personas velarizadas forman una especie de L tumbada invertida. En este estudio trataremos por simplicidad solo el primer grupo, ya que las formas del tema de perfecto tanto del segundo grupo como del tercero poseían una velar etimológica en el catalán medieval (3 PLĀNXIT > planc 'plañó', 3 CRĒVUIT > crec 'creció'), y no analógica como en el caso de 3 DīxIt $>$ dix $>>$ diguée 'dijo'2.

\begin{tabular}{lcccccc}
\hline \multicolumn{7}{c}{ TABLA 2. TEMA DE PRESENTE EN CATALÁN ANTIGUO DE } \\
\hline & 1 & 2 & 3 & 4 & 5 & 6 \\
\hline Pres. ind. & dic- $\varnothing$ & diu-s & diu & dei- $m$ & dei-ts & di-en \\
\hline Pres. subj. & dig- $a$ & digu-es & dig- $a$ & dig-am & dig-au & digu-en \\
\hline
\end{tabular}

\begin{tabular}{lcccccc}
\hline \multicolumn{5}{c}{ TABLA } & 3. TEMA DE & PRESENTE EN CATALÁN ANTIGUO DE PLÀNYER 'PLAÑIR' \\
\hline & 1 & 2 & 3 & 4 & 5 & 6 \\
\hline Pres. ind. & planc- $\varnothing$ & plany-s & plany & plany-em & plany-eu & plany-en \\
\hline Pres. subj. & plang- $a$ & plangu-es & plang-a & plang-am & plang-au & plang-uen \\
\hline
\end{tabular}

2 Con el signo > indicamos las formas resultantes de la evolución del latín al catalán, mientras que con el signo $>>$ señalamos las que son producto de cambios analógicos. 
TABLA 4. TEMA DE PRESENTE EN CATALÁN ANTIGUO DE CRÉIXER 'CRECER’

\begin{tabular}{lcccccc}
\hline & 1 & 2 & 3 & 4 & 5 & 6 \\
\hline Pres. ind. & cresc- $\varnothing$ & creix-es & creix & creix-em & creix-eu & creix-en \\
\hline Pres. subj. & cresc- $a$ & cresqu-es & cresc- $a$ & cresc-am & cresc-au & cresqu-en \\
\hline
\end{tabular}

Por lo que respecta al tema de perfecto, el grupo más numeroso de verbos con formas velarizadas estaba constituido por aquellos que presentaban en latín clásico un perfecto fuerte en -Uĩ: DEBERE (DEBUIT), PLACERE (PLACUIT), etc., o que adoptaron este modelo en latín vulgar: CREDERE, BIBERE, etc. El origen de la velar en estos casos no está tan claro como en el tema de presente. Para la mayoría de autores (Coromines 1971: 324, Meyer-Lübke 1895: \$278, Moll 2006: 210, Pérez Saldanya 1998: 230-231 y Querol 2011: 30), la velar deriva del refuerzo de la desinencia aspectual de los prefectos fuertes latinos acabados en -Uĩ: se considera que la semivocal /w/ de los tiempos perfectivos se reforzó y se convirtió en una labiovelar obstruyente: $w>g^{w}>g$. Sin embargo, Wheeler (2011: 195-198) considera errónea esta evolución y propugna la que propuso para el occitano Ronjat (1937: \$570), para quien la velar surge como una extensión analógica desde un pequeño grupo de verbos (PLACUit > plac 'plació') en que se sabe a ciencia cierta que la velar era etimológica; en esta interpretación, la creación analógica de perfectos velarizados en casos como DEBERE serviría para evitar el sincretismo de tiempo verbal que se hubiese producido entre las personas $3 .^{a}$ y $4 .^{a}$ del presente de indicativo y del pretérito perfecto simple ${ }^{3}$.

Dado que esta cuestión no es el objeto de estudio que nos ocupa en este trabajo, no nos extenderemos más en esta discusión, puesto que, con independencia de la propuesta, en los dos casos la consonante velar se comportaba morfológicamente como una marca de perfecto. Así pues, la consonante velar aparecía en los siguientes tiempos perfectivos: pretérito perfecto simple, pluscuamperfecto de indicativo y pluscuamperfecto de subjuntivo. Esta distribución ha recibido el nombre de patrón PyTA (Maiden 2018: 48), acrónimo de perfecto y tiempos afines. En la tabla 5 adjuntamos el paradigma velarizado del tema de perfecto o patrón PyTA en catalán. Presentamos solamente la tercera persona, ya que en estos tiempos todas las personas presentan el afijo velar.

\begin{tabular}{ll}
\hline \multicolumn{2}{|c|}{ TABLA 5. EL SEGMENTO VELAR EN EL TEMA DE PERFECTO } \\
\hline & Velar etimológica: plaure 'placer') \\
\hline Pretérito perfecto simple & 3 plac > plagué \\
\hline Pluscuamperfecto de indicativo & 3 plagra $>$ plaguera \\
\hline Pluscuamperfecto de subjuntivo & 3 plagués \\
\hline
\end{tabular}

${ }^{3}$ Por ejemplo, la homofonía que se hubiese producido entre la $3 .{ }^{a}$ persona del presente de indicativo de deure, DĒBET > deu 'debe', y la de un hipotético pretérito perfecto simple sin velarizar, DĒBUIT > *DĒB(U)IT > *deu 'debió'. 
Cabe destacar que la presencia de la velar en el patrón PyTA es una característica que distingue al catalán, y también al occitano, dentro de las lenguas románicas (Wheeler 2011: 194). En contraste con estas dos lenguas, en otra lengua románica próxima al catalán como el español, el afijo velar se circunscribe únicamente al patrón $\mathrm{L}$, esto es, a la primera persona del presente de indicativo y a todas las personas del presente de subjuntivo, con diferentes subgrupos (O'Neill 2015: 490): verbos en los que la velar es sonora, como tener ( 1 tengo; 1 tenga); verbos en que es sorda, como crecer ( 1 crezco; 1 crezca), y verbos cuyo radical acaba en vocal y la velar sonora va precedida de una yod [j], como caer (1 caigo; 1 caiga).

Recapitulando, hemos visto que la velar etimológica, en unos casos, formaba parte del segmento final radical y, en otros, era una marca del tema de perfecto. En ambos casos, se observa en la lengua una tendencia a extender las formas velarizadas más allá de su ámbito original. Nuestro objetivo en los siguientes apartados del trabajo es, en primer lugar, relacionar la expansión de este afijo con un tipo especial de reanálisis: la exaptación (sección 2), y, en segundo lugar, aportar luz sobre la expansión de la consonante velar, desde el siglo XIII al Xv, en tres verbos que, como veremos, representan tres de las subclases a las que se ha ido extendiendo la velar: dir, deure y prendre (sección 3).

\section{LA REUTILIZACIÓN DEL AFIJO VELAR COMO MARCA DE CLASE: EXAPTACIÓN}

Dentro de las teorías del cambio lingüístico, el proceso de expansión que experimentó el afijo velar parece seguir el patrón típico de los casos que se recogen bajo el concepto de exaptación. Lass (1990) es el primero que introdujo en el campo de la lingüística este concepto, proveniente de la biología evolutiva (Gould y Vrba 1982). Lass, concretamente, sugirió emplear el término de exaptación metafóricamente, para tratar algunos cambios que se producen en los márgenes de los sistemas lingüísticos. Según Lass (1990: 82), cuando una forma pierde su función, o solo es marginal dentro de un sistema lingüístico, existen tres posibilidades:

a) se puede perder;

b) se puede mantener como una forma marginal o no funcional (supleción, irregularidad $)^{4}$;

c) se puede mantener, pero, en vez de relegarla, se reutiliza con otra función, que puede ser igualmente sistemática.

${ }^{4}$ Este caso podemos relacionarlo con lo que le ha sucedido al afijo velar en espańol, puesto que hoy en día las formas velarizadas solo aparecen en el patrón L, y en un número de verbos bastante reducido. Sin embargo, en castellano antiguo, la nómina de verbos que contaban con la consonante velar era mayor (O’Neill 2015: 492). 
La tercera opción, el reciclaje de material morfológico no funcional (junk 'basura', en la terminología de Lass) que, al reutilizarse, consigue un nuevo estatus funcional, es lo que constituye la exaptación en el cambio lingüístico.

En un trabajo más reciente, Wall y Octavio de Toledo (2016: 343-344) proponen restringir el concepto de exaptación a los cambios lingüísticos en los que alguna forma o estructura desarrolla una nueva función inesperada, en el sentido de que se desvía de un patrón típicamente observado de gramaticalización avanzada. Wall y Octavio de Toledo subrayan que esta clase de cambios necesitan un nombre dentro de la teoría del cambio lingüístico, ya que son bastante más frecuentes de lo que se presupone. De esta manera, recogerlos bajo la categoría de exaptación ayudaría a identificar el conjunto de cambios que no encajan en la descripción de los procesos arquetípicos de gramaticalización.

Por otra parte, la exaptación ha sido comparada también con el reanálisis. Harris y Campbell (1995: 61) definen el reanálisis como un «mecanismo que cambia la estructura subyacente de un patrón sintáctico y que no implica ninguna modificación inmediata o intrínseca de su manifestación superficial». En el caso de la exaptación del afijo velar, en su reciclaje para otras funciones, hay reanálisis, ya que un constituyente asume una función distinta, asociada a una posición diferente dentro de la jerarquía de la palabra: de ser una marca de flexión con el valor semántico de perfectividad, y ocupar una posición más externa, se convierte en una marca de clase verbal sin ese valor inicial y pasa a ocupar una posición más interna.

El proceso de pérdida de la asociación entre la velar y la perfectividad es gradual. Pérez Saldanya (1996: 285) propone una de las claves que posibilitaron la expansión de la velar a contextos en que no era propia: la motivación semántica del afijo velar y la estructuración icónica del sistema de perfecto velarizado empezó a desdibujarse cuando las perífrasis con HABEO/sUM + participio asumieron el valor de perfectividad de las antiguas formas sintéticas en /-ra-/ y /-s/. De esta manera, el pluscuamperfecto de indicativo latino en $r a-$ : volueram > volgra 'había querido', empezó a perder el valor perfectivo y a convertirse en un condicional simple, con el sentido de 'querría', hacia el siglo XIV. El pluscuamperfecto de subjuntivo latino: VOLUISSEM > volgués 'hubiese querido', a su vez, empezó a perder también el valor de perfectividad y a funcionar como imperfecto de subjuntivo, con el sentido de 'quisiera', en esta misma época.

Esta pérdida de motivación semántica del segmento velar posibilitó que la consonante velar se generalizara analógicamente en formas claramente imperfectivas como las del sistema de presente, es decir, presente de indicativo (1 bec 'bebo') y presente de subjuntivo (3 bega 'beba'). Esta expansión se vio favorecida por la existencia, como hemos comentado antes, de un conjunto de verbos como dir o dur que presentaban la velar en el tema de presente (verbos del patrón L), en los que formaba parte del segmento final del radical.

En definitiva, la expansión de la velar parece encajar dentro de las propiedades esperables en los procesos de exaptación, ya que un segmento que, en un primer momento, tenía el valor semántico de perfecto acabó reutilizándose como marca de clase verbal. Como consecuencia de la pérdida de ese mismo valor, el proceso de velarización afectó incluso a verbos que no presentaban la consonante velar 
ni en el tema de presente ni en el de perfecto, como, por ejemplo, prendre 'tomar' o vendre 'vender's.

\section{EXTENSIÓN DE LA VELAR ENTRE LOS SIGLOS XIII Y XV}

En esta sección, en primer lugar, veremos el corpus que se ha utilizado para extraer las formas verbales que son objeto de estudio, y, en segundo lugar, pasaremos al análisis de las formas extraídas para ver el estadio de evolución que presenta el afijo velar entre los siglos xiII y xv. Para ello, como ya se ha mencionado al comienzo del artículo, hemos elegido tres verbos paradigmáticos de cada uno de los subgrupos en los que se fue extendiendo la velar: dir 'decir', deure 'deber' y prendre 'tomar'.

\subsection{Corpus}

Por lo que respecta a la obtención de datos, para la extracción de formas verbales hemos creado un corpus formado por obras de la segunda mitad de los siglos XIII, XIV y XV, con el objetivo de ver qué estado de evolución presentaban las formas verbales hacia el final de cada siglo. Por cada centuria contamos con 180000 palabras: 90000 palabras corresponden a obras del catalán occidental, y otras tantas, a obras del catalán oriental, con el objetivo de que los dos grandes bloques que forman esta lengua estén igualmente representados. Cabe añadir que en algún caso no hemos obtenido datos suficientes para elaborar un análisis consistente, por lo que hemos recurrido al Corpus Informatitzat del Català Antic (a partir de ahora, CICA), para comprobar la tendencia general en el resto de obras del mismo periodo. Las obras que forman nuestro corpus son:

- Siglo XIII: Costums de Tortosa y Llibre de contemplació en Déu.

- Siglo XIV: Epistolari de la València Medieval (I), Els manuals de consells medievals de Xàtiva (1376-1380), Corbatxo, Els quatre llibres de la reina Elionor de Sicilia, La fiyla del rey d'Ungria y Lo somni.

- Siglo XV: Tirant lo Blanch, Historries e conquestes del realme d'Aragó e principat de Catalunya y Llibre de les solemnitats de Barcelona.

- Corpus Informatitzat del Català Antic (2010), Joan Torruella (dir.), con la colaboración de Manuel Pérez Saldanya y Josep Martines.

5 En estos casos, la introducción de la velar permitía resolver también problemas de homonimia entre la $1 .^{a}$ y la $3 .^{a}$ persona en el presente de indicativo, que originalmente presentaban la misma forma: pren; la forma analógica prenc para la $1 .{ }^{a}$ persona permite diferenciarla de la $3 .^{a}$ persona pren. 


\subsection{Verbos con velar etimológica en el tema de presente (patrón L)}

El primer verbo que analizamos es dir, que, como hemos dicho, forma parte del grupo de verbos en los que la velar era etimológica en el tema de presente, ya que formaba parte del segmento final del radical: DİCO > dic 'digo', DİCAT > diga 'diga' (patrón L). En este caso, lo que nos interesa es estudiar la extensión de la velar al tema de perfecto, en que no era etimológica, de modo que se pasa de un patrón $\mathrm{L}$ a un patrón PyTA. Para mostrar este cambio, analizamos solamente los datos del pretérito perfecto simple y del imperfecto de subjuntivo, puesto que no hemos obtenidos suficientes datos del condicional para poder hacer un análisis consistente. En la tabla 6 recogemos las formas del pretérito perfecto simple, velarizadas y no velarizadas, que hemos extraído del corpus.

\begin{tabular}{|c|c|c|c|c|c|c|c|c|c|c|}
\hline \multicolumn{11}{|c|}{ TABLA 6. PRETÉRITO PERFECTO SIMPLE DE DIR 'DECIR' } \\
\hline \multicolumn{3}{|c|}{ SigLO XIII } & \multicolumn{4}{|c|}{ SigLO XIV } & \multicolumn{4}{|c|}{ SigLO XV } \\
\hline \multicolumn{2}{|c|}{-velarizado } & \multirow{2}{*}{ +velarizado } & \multicolumn{2}{|c|}{-velarizado } & \multicolumn{2}{|c|}{ +velarizado } & \multicolumn{2}{|c|}{-velarizado } & \multicolumn{2}{|c|}{ +velarizado } \\
\hline Formas & Casos & & Formas & Casos & Formas & Casos & Formas & Casos & Formas & Casos \\
\hline 1 dix & & & $3 d i x$ & 252 & 1 diguí & 86 & $3 d i x$ & 526 & 1 diguí & 4 \\
\hline \multirow[t]{2}{*}{$3 d i x$} & 10 & & 4 dixem & 5 & 4 diguem & & & & 6 digueren & 65 \\
\hline & & & 6 dixeren & 21 & 6 digueren & 5 & & & & \\
\hline Total & 11 & & Total & 278 & Total & 92 & Total & 526 & Total & 69 \\
\hline Porc. & $100 \%$ & & Porc. & $75,14 \%$ & Porc. & $24,86 \%$ & Porc. & $88,4 \%$ & Porc. & $11,6 \%$ \\
\hline
\end{tabular}

Como se observa en la tabla, en el siglo XiII solo se documentan formas con sibilante, que son las etimológicas: 1 DİXI $>$ dix 'dije', 3 DīXIT > dix 'dijo' (v. el ejemplo de (1) $)^{6}$. Hay que esperar hasta el siglo XIV para encontrar formas velarizadas. En este siglo, podemos ver grandes diferencias entre personas: mientras que en la tercera persona se sigue conservando la forma etimológica, en la primera persona ya solo aparece la forma innovadora velarizada (v. (2)). Una de las ventajas de la introducción de esta nueva forma es que permitía deshacer el sincretismo formal entre la primera y la tercera persona, representadas ambas como dix anteriormente.

(1) [...] jo trobe aital hom e axí com vós avíetz jutyat dix-li e fiu-li saber si volia acórrer a aital peynora que avia assignada a sson creedor [...] (Costums de Tortosa, p. 31)

(2) -Com, l'esperit? - diguí jo-. No puch creure que l'esperit sia res ne puxe tenir altre camí sinó aquell que la carn té. (Lo somni, p. 58)

${ }^{6}$ En los Costums de Tortosa y en el Llibre de contemplació en Déu no hemos encontrado formas velarizadas de este tiempo. En el CICA, en cambio, sí que hemos encontrado formas con velar del pretérito perfecto simple ya en la segunda mitad del siglo xiII, como 3 digué o 6 digueren. Sin embargo, en este trabajo basamos el análisis únicamente en las formas que hemos extraído de nuestro corpus. 
Por lo que respecta a las otras dos personas de las que tenemos datos, la cuarta y la sexta, vemos que también se han introducido las formas innovadoras (v. (3)), si bien las etimológicas (v. (4)) son aún mayoritarias.

(3) altres digueren que una part del cervell tenia lo principat de la ànima; altres, que.l loch e cadira de la ànima eren en lo cor. (Lo somni, p. 72)

(4) Et los dits official e vicari dixeren que ells parlarien del dit feyt ab los clergues de la dita ciutat e parlat ab aquells els retrien resposta als jurats de la dita ciutat de ço que acordarien. (Manual de consells 1378-1379, p. 117)

Los datos del siglo xv son similares a los del siglo anterior: nuevamente, solo se encuentran formas de la primera persona velarizadas, mientras que en la tercera la forma con sibilante, sin velarizar, sigue siendo la única. La diferencia principal respecto del siglo anterior es que en la sexta persona la forma innovadora se ha acabado de imponer totalmente. En un cómputo global, vemos que la tercera persona es la más reticente al cambio, quizás por su alta frecuencia de uso.

Por lo que atañe al imperfecto de subjuntivo, las formas de este tiempo verbal provienen del pluscuamperfecto de subjuntivo latino: DİXISSET $>$ dixés $>>$ digués 'dijese'. Estas formas, como hemos mencionado anteriormente, fueron perdiendo el valor de perfectividad y asumiendo el valor modal que tienen en la actualidad. En cuanto a los datos extraídos, en la tabla 7 vemos que, a diferencia del pretérito perfecto simple, en el siglo XIII ya se encuentran en el corpus formas velarizadas (v. (5)) y que, además, aparecen con la misma frecuencia que las etimológicas (v. (6)). En el siglo XIV, del que disponemos de más datos que del siglo anterior, las formas velarizadas superan ligeramente en número a las formas con sibilante. Además, para las personas primera, segunda y quinta solamente encontramos formas innovadoras. Por último, en el xv, todas las formas que hemos extraído son ya velarizadas. Si seguimos con la tesis de que la frecuencia de uso condiciona el ritmo de generalización de las formas analógicas, la baja frecuencia del imperfecto de subjuntivo seguramente ha favorecido la rápida extensión del afijo velar.

\begin{tabular}{|c|c|c|c|c|c|c|c|c|c|c|}
\hline \multicolumn{11}{|c|}{ TABLA 7. IMPERFECTO DE SUBJUNTIVO DE DIR 'DECIR' } \\
\hline \multicolumn{4}{|c|}{ Siglo XIII } & \multicolumn{4}{|c|}{ Siglo XIV } & \multicolumn{3}{|c|}{ SigLo XV } \\
\hline \multicolumn{2}{|c|}{-velarizado } & \multicolumn{2}{|c|}{ +velarizado } & \multicolumn{2}{|c|}{-velarizado } & \multicolumn{2}{|c|}{ +velarizado } & \multirow[t]{2}{*}{-velarizado } & \multicolumn{2}{|c|}{ +velarizado } \\
\hline Formas & Casos & Formas & Casos & Formas & Casos & Formas & Casos & & Formas & Casos \\
\hline \multirow[t]{5}{*}{3 dixés } & 2 & 3 digués & 2 & 3 dixés & 6 & 1 digués & 5 & & 3 digués & 14 \\
\hline & & & & 6 dixessen & 7 & 2 diguesses & & & 5 diguésseu & 2 \\
\hline & & & & & & 3 digués & 7 & & 6 diguessen & 5 \\
\hline & & & & & & 5 diguéssets & & & & \\
\hline & & & & & & 6 diguessen & 2 & & & \\
\hline Total & 2 & Total & 2 & Total & 13 & Total & 16 & & Total & 21 \\
\hline Porc. & $50 \%$ & Porc. & $50 \%$ & Porc. & $44,83 \%$ & Porc. & $55,17 \%$ & & Porc. & $100 \%$ \\
\hline
\end{tabular}


(5) Però si l'ostaler pot provar que él digés a aquela persona qui la cosa aja perduda, ans que la perdés, que, si tenia alcuna cosa que fes a gardar, que í la í liuràs. (Costums de Tortosa, p. 125)

(6) Veritat demaná, Seyner, a la mia anima que dixés d'on li ve que ela cuyda esser bona per si metexa e cuyda aver en si noblesa e vertut, la qual no y es enaxí con se cuyda. (Llibre de contemplació en Déu, p. 232)

\subsection{Verbos con velar etimológica en el tema de perfecto (patrón PýTA)}

El segundo verbo objeto de análisis es deure, que forma parte del conjunto de verbos en que la velar era etimológica en el tema de perfecto (patrón PyTA), pero no en el de presente (patrón L). En este subgrupo, por lo tanto, nos interesa analizar la extensión de la velar a las formas de presente. En este tiempo, las formas etimológicas no responden a un único patrón, sino que presentan varias soluciones, como ilustramos con los ejemplos de (7): en la $1 .{ }^{a}$ persona del presente de indicativo de los verbos deure y valdre 'valer', deig (7a) o vall (7b), la forma originaria acababa en una consonante palatal, $[\mathrm{t} f]$ y $[K]$, respectivamente, mientras que en la $1 .^{a}$ persona del verbo moure 'mover', mou (7c), lo hacía en una semivocal [w]. Sustituir todas estas terminaciones por una consonante velar, como en las formas dec, valc y moc de (7), sirvió para dotar de una mayor uniformidad y estabilidad a esta clase de verbos (Pérez Saldanya 1998: 79).

$$
\begin{array}{ll}
\text { a. } & \text { DĒBEŌ }>\text { deig }>>\text { dec 'debo' } \\
\text { b. } & \text { VALEŌ }>\text { vall }>\text { valc 'valgo' } \\
\text { c. } & \text { MOVEŌ }>\text { mou }>>\text { moc 'muevo' }
\end{array}
$$

El primero de los tiempos que analizamos es el presente de indicativo (v. tabla 8). En primer lugar, vemos que en el siglo XIII aún se mantiene la forma etimológica de la 1 . $^{a}$ persona: $\operatorname{deig}(8)$, sin presencia aún de formas velarizadas. No es hasta el siglo XIV cuando aparece la primera variante con el afijo velar (9). En términos numéricos, el cambio más significativo se produce en el paso del XIV al XV, puesto que en este último siglo predominan las formas velarizadas.

\begin{tabular}{lccccccccc}
\hline \multicolumn{7}{c}{ TABLA 8. PRESENTE DE INDICATIVO DE DEURE 'DEBER' } \\
\hline \multicolumn{7}{c}{ Siglo XIII } & \multicolumn{5}{c}{ Siglo XIV } & \multicolumn{3}{c}{ Siglo XV } \\
\hline \multicolumn{2}{c}{-velarizado } & +velarizado & -velarizado & +velarizado & -velarizado & \multicolumn{2}{c}{ +velarizado } \\
\hline Formas & Casos & Formas & Casos & Formas & Casos & Formas & Casos & Formas & Casos \\
1 deig & 9 & 1 deig & 3 & 1 dec & & 1 deig & 2 & 1 dec & 8 \\
Total & 9 & Total & 3 & Total & 1 & Total & 2 & Total & 8 \\
Porc. & $100 \%$ & Porc. & $75 \%$ & Porc. & $25 \%$ & Porc. & $20 \%$ & Porc. & $80 \%$ \\
\hline
\end{tabular}


(8) Esdevén-se a vegades que hom compra d'altre la sua cosa metexa; on és raon que si jo lo preu e pagat a altre per la mia cosa metexa, que.l deg cobrar. (Costums de Tortosa, p. 187)

(9) E per so que asò se.n saguescha, devotament prech Aquell del qual so que yo dech dir e tot altre bé és proceyt e proceex. (Corbatxo, p. 10)

El segundo tiempo que tratamos es el presente de subjuntivo (v. tabla 9). Al igual que ocurre con el presente de indicativo, debemos esperar hasta el siglo XIV para encontrar formas velarizadas (10), ya que en el siglo XIII solo se documentan formas palatalizadas (11). Del xv no hemos obtenido ninguna forma velarizada. Para confirmar si esta tendencia es la general en el resto de las obras de la segunda mitad de dicho siglo, hemos consultado la documentación del CICA. Los datos obtenidos de esta consulta difieren bastante de los de nuestro corpus: así, de la forma velarizada de 3. ${ }^{a}$ persona dega se encuentran 32 casos, frente a los 25 de la forma con palatal deja. Del mismo modo, hemos encontrado 7 casos de la $6 .{ }^{a}$ persona velarizada deguen frente a los 6 de la forma con palatal degen. Por lo tanto, la tendencia general durante la segunda mitad de siglo era la expansión de la consonante velar, que ya aparece más veces que las formas etimológicas, como sucedía con la $1 .^{a}$ persona del presente de indicativo en ese mismo siglo (v. tabla 6).

\begin{tabular}{|c|c|c|c|c|c|c|c|c|c|}
\hline \multicolumn{10}{|c|}{ TABLA 9. PRESENTE DE SUBJUNTIVO DE DEURE 'DEBER' } \\
\hline \multicolumn{3}{|c|}{ Siglo XIII } & \multicolumn{4}{|c|}{ SigLo XIV } & \multicolumn{3}{|c|}{ SIgLo XV } \\
\hline \multicolumn{2}{|c|}{-velarizado } & \multirow[t]{2}{*}{ +velarizado } & \multicolumn{2}{|c|}{-velarizado } & \multicolumn{2}{|c|}{ +velarizado } & \multicolumn{2}{|c|}{-velarizado } & \multirow[t]{2}{*}{ +velarizado } \\
\hline Formas & Casos & & Formas & Casos & Formas & Casos & Formas & Casos & \\
\hline 1 deja & 2 & & 1 deja & 2 & 1 dega & & 1 deja & & \\
\hline 3 deja & 40 & & 3 deja & 23 & 2 degues & & 3 deja & & \\
\hline 4 dejam & 2 & & 4 dejam & & 6 deguen & 2 & 4 dejam & & \\
\hline 5 dejatz & 8 & & 5 dejats & 2 & & & 6 degen & & \\
\hline 6 degen & 11 & & 6 degen & 4 & & & & & \\
\hline Total & 63 & & Total & 32 & Total & 4 & Total & 4 & \\
\hline Porc. & $100 \%$ & & Porc. & $88,89 \%$ & Porc. & $11,11 \%$ & Porc. & $100 \%$ & \\
\hline
\end{tabular}

(10) -Ço que t'hé dit ver és -dix ell-, e no crech pas que.t degues clamar de fortuna [...] (Lo somni, p. 230)

(11) [...] cor enaxí, Seyner, con vos avetz ordenat que home deja amar vos ab la primera entenció e si metex ab la segona [...] (Llibre de contemplació en Déu, p. 200)

\subsection{Verbos Sin Velar etimológica en NiNGUNo de LOS DOS temas}

Por último, el tercer subgrupo analizado está formado por verbos en que la velar no era etimológica ni en el tema de presente ni en el de perfecto. Dentro de este conjunto, encontramos un amplio número de verbos que tenían el afijo $s$ como marca de perfecto (12), bien por etimología, como en el verbo remandre 'permane- 
cer' (12a), o bien por analogía, como en los verbos defendre 'defender' (12c) y prendre 'tomar' (12b), utilizado para ilustrar la extensión de la velar en esta subclase, y que etimológicamente solamente presentaba la sibilante en el participio: PRĒNSÚ > pres 'tomado'.
a. REMĀNSIT > remàs 'permaneció'
b. PRĒNDIT >> pres 'tomó'
c. DĒFENDIT $\gg$ defés 'defendió'

Dentro del tema de presente, discutiremos solamente el presente de subjuntivo, ya que no se han obtenido datos suficientemente representativos del indicativo (v. tabla 10). Así, vemos que ya en el siglo XIII las formas velarizadas (13) superan a las etimológicas (14). Este predominio de las formas analógicas es, cuando menos, sorprendente, puesto que, como ya se ha mencionado, se trata de verbos sin una velar etimológica en los otros dos temas que pudiera haber favorecido la expansión, como ocurre en las otras dos subclases analizadas en los epígrafes anteriores. Los siglos XIV y XV son una mera continuación de las tendencias del siglo XIII, de manera que en el último siglo todas las formas extraídas del corpus presentan ya el afijo velar. Esto significaría que, ya en el siglo xv, y probablemente en el xIv, la velar hubiese perdido completamente el valor de perfectividad puesto que, si no, no se entiende esta rápida expansión en el tema de presente.

\begin{tabular}{|c|c|c|c|c|c|c|c|c|c|c|}
\hline \multicolumn{11}{|c|}{ TABLA 10. PRESENTE DE SUBJUNTIVO DE PRENDRE 'TOMAR' } \\
\hline \multicolumn{4}{|c|}{ Siglo XIII } & \multicolumn{4}{|c|}{ Siglo XIV } & \multicolumn{3}{|c|}{ Siglo XV } \\
\hline \multicolumn{2}{|c|}{-velarizado } & \multicolumn{2}{|c|}{ +velarizado } & \multicolumn{2}{|c|}{-velarizado } & \multicolumn{2}{|c|}{ +velarizado } & \multirow[t]{2}{*}{-velarizado } & \multicolumn{2}{|c|}{ +velarizado } \\
\hline Formas & Casos & Formas & Casos & Formas & Casos & Formas & Casos & & Formas & Casos \\
\hline 3 prena & 8 & 3 prenga & 14 & 3 prena & & 1 prenga & & & 2 prengues & \\
\hline 4 prenam & 2 & 6 prenguen & 7 & 5 prenats & & 2 prengues & & & 3 prenga & \\
\hline \multirow[t]{3}{*}{6 prenen } & 2 & & & & & 3 prenga & 2 & & 4 prengam & \\
\hline & & & & & & 5 prengats & & & 5 prengau & 3 \\
\hline & & & & & & 6 prenguen & 4 & & & \\
\hline Total & 12 & Total & 21 & Total & 2 & Total & 9 & & Total & 6 \\
\hline Porc. & $36,36 \%$ & Porc. & $63,64 \%$ & Porc. & $18,18 \%$ & Porc. & $81,82 \%$ & & Porc. & $100 \%$ \\
\hline
\end{tabular}

(13) que.ls troba fora de l'alberch que.l deutor que él los prenga e.ls liure al creedor. (Costums de Tortosa, p. 30)

(14) Per aytal manera que hom prena, Seyner, en si metex que a vos conega. (Llibre de contemplació en Déu, p. 297)

En cuanto a las formas de perfecto, el radical del tema de perfecto del verbo prendre anterior a la generalización de la velar contenía una sibilante: pres 'tomó' (v. (12)). Cabe remarcar que, como hemos indicado antes, esta tampoco era la forma etimológica de este verbo, sino que se trata de una forma analógica. Nos encontramos, pues, ante una sustitución de una forma que ya había sustituido a la variante 
original. Por lo que respecta a la extensión de la velar, en el pretérito perfecto simple (v. tabla 11) debemos esperar hasta el siglo XIV para encontrar formas velarizadas (v. (15)). Las formas con sibilante (v. (16)) siguen siendo las mayoritarias en ese siglo, si bien es cierto que ya solamente se documentan formas velarizadas de $1 .{ }^{a}$ persona; en el caso de la $6 .^{a}$, aunque hay ejemplos con sibilante y ejemplos con velar, estos últimos son ya más comunes. Por último, en el xv, aunque en el cómputo global las formas con sibilante siguen siendo las más abundantes, vemos que la única persona en la que resisten es la $3 .^{a}$, que es la más utilizada. Nuevamente observamos que la frecuencia incide en el mantenimiento de las formas no analógicas: al ser las formas más usadas, son las más reticentes al cambio.

\begin{tabular}{|c|c|c|c|c|c|c|c|c|c|c|}
\hline \multicolumn{3}{|c|}{ Siglo XIII } & \multicolumn{4}{|c|}{ Siglo XIV } & \multicolumn{4}{|c|}{ SigLo XV } \\
\hline -velari & zado & +velarizado & -velari & zado & +velariza & ado & -vela & izado & +velariz: & zado \\
\hline Formas & Casos & & Formas & Casos & Formas & Casos & Formas & Casos & Formas & Casos \\
\hline $\begin{array}{l}3 \text { pres } \\
6 \text { preseren }\end{array}$ & 2 & & $\begin{array}{l}3 \text { pres } \\
4 \text { presem } \\
6 \text { preseren }\end{array}$ & 25 & $\begin{array}{l}1 \text { prengui } \\
6 \text { prengueren }\end{array}$ & n 4 & 3 pres & 285 & $\begin{array}{l}1 \text { prengui } \\
3 \text { prengué } \\
6 \text { prenguere }\end{array}$ & $\begin{array}{c}4 \\
\text { en } 66\end{array}$ \\
\hline $\begin{array}{l}\text { Total } \\
\text { Porc. }\end{array}$ & $\begin{array}{c}3 \\
100 \%\end{array}$ & & $\begin{array}{l}\text { Total } \\
\text { Porc. }\end{array}$ & $\begin{array}{c}27 \\
84,38 \%\end{array}$ & $\begin{array}{l}\text { Total } \\
\text { Porc. }\end{array}$ & $\begin{array}{c}5 \\
15,62 \%\end{array}$ & $\begin{array}{l}\text { Total } \\
\text { Porc. }\end{array}$ & $\begin{array}{c}285 \\
80,06 \%\end{array}$ & $\begin{array}{l}\text { Total } \\
\text { Porc. }\end{array}$ & $\begin{array}{c}71 \\
19,94 \%\end{array}$ \\
\hline
\end{tabular}

(15) ab lo comte de Medina e ab lo Rexon, vengren córrer al termenal de Terol e feren aquí assats de dan, e assajaren de combatre Sarrió e prengueren-hi dan. (Epistolari de la València Medieval (I), p. 210)

(16) e les parques Cloto, Làchesis e Àntropos, per la dolçor del meu cant, havent pietat de mi, se preseren a plorar e, ensemps, cessaren exerçir lurs officis. ( $L o$ somni, p. 164)

Por otra parte, en el imperfecto de subjuntivo (v. tabla 12), ya encontramos una forma velarizada en el siglo XIII (v. (17)), aunque sigue habiendo mayoría de formas con sibilante. En el siglo siguiente hay un cambio brusco, ya que las formas con velar pasan a ser preponderantes. El siglo Xv supone una continuación del anterior, dado que las formas innovadoras siguen aumentando hasta el punto de que solamente se documenta un caso sin velarizar (v. (18)). Constatamos, además, la misma situación que habíamos observado para el tema de perfecto del verbo dir: mientras que en el pretérito perfecto simple cuesta más encontrar formas velarizadas y estas siguen siendo minoría con el paso de los siglos, en el imperfecto de subjuntivo las formas con la consonante velar se introducen ya en el siglo xiII y pasan a ser rápidamente las variantes mayoritarias. Parece que, como en el verbo dir, la menor frecuencia de uso del imperfecto de subjuntivo ha favorecido la rápida penetración de la velar. 


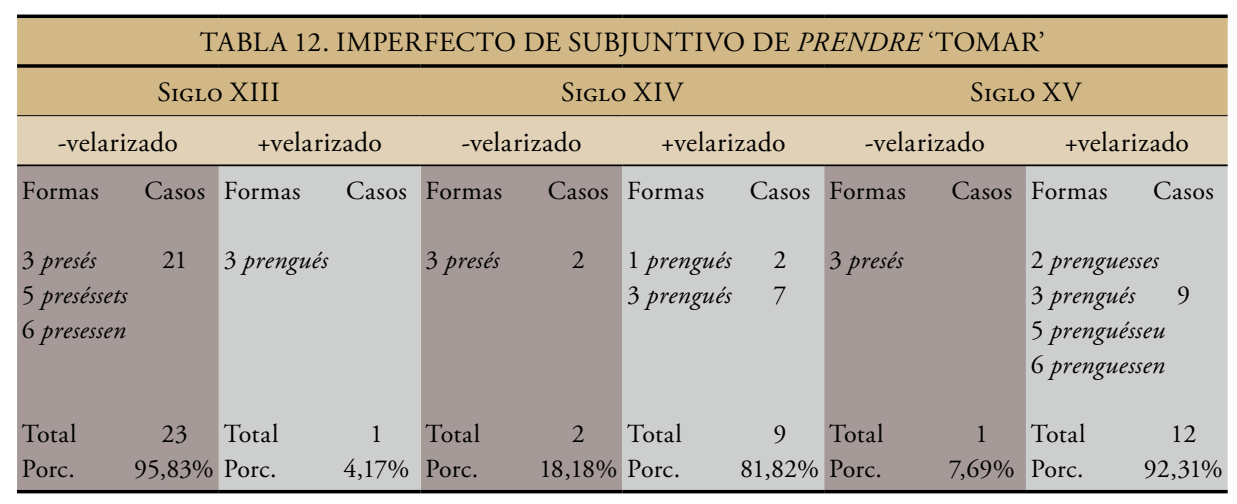

(17) Però si lo dit emphiteota, dintre aquels tres ayns, volria pagar lo cens, e el seynor no.l volia pendre o ç'absentava perquè no.l prengés ne l'inphiteota lo y pogés donar ne pagar. (Costums de Tortosa, p. 261)

(18) deu-se e-s pot mitigar tota lamentació e efusió de làgrimes, les quals may suppliren en cessar la mort, e deu-sa resumir spirit de consolació, com presés primer los sagraments ecclesiàstichs, ço és, d'armadura celestial. (Llibre de les solemnitats de Barcelona, p. 207)

\section{CONCLUSIONES}

A lo largo de este estudio hemos repasado la evolución de la subclase de verbos velarizados que se incluye dentro de la segunda conjugación del catalán. De este modo, se han analizado las distintas procedencias del afijo velar, que en un grupo de verbos procede del tema de presente latino y, en otros, era una marca de perfecto. En el primer grupo, la velar formaba parte del segmento final radical y no tenía ningún valor semántico, y las formas velarizadas adoptaban la distribución del patrón $\mathrm{L}$ (es decir, $1 .{ }^{a}$ persona del presente de indicativo: 1 dic, y presente de subjuntivo: 3 diga). En el segundo grupo, la velar tenía un valor semántico de perfectividad en un primer momento y se distribuía según el patrón PyTA (es decir, pretérito perfecto simple: 3 degué; pluscuamperfecto de indicativo: 3 deguera, y pluscuamperfecto de subjuntivo: 3 degués). Con el paso del tiempo el valor de perfectividad se fue perdiendo, como muestra que el pluscuamperfecto de indicativo pasara a ser el condicional y el pluscuamperfecto de subjuntivo, el imperfecto del mismo modo.

Por lo que respecta a los datos extraídos del corpus, hemos tomado como referencia tres verbos, representativos de tres subclases diferentes: dir, con velar etimológica en el tema de presente; deure, con velar etimológica en el tema de perfecto, y prendre, sin velar etimológica en ninguno de los dos temas. Una vez completado el análisis, constatamos ciertas similitudes entre algunos tiempos. Así, si prestamos atención a los temas de perfecto de dir y prendre, podemos ver cómo el comportamiento en el pretérito perfecto simple y el imperfecto de subjuntivo de ambos verbos es prácticamente paralelo. En efecto, en el primero de los tiempos no encontramos 
formas velarizadas para ninguno de los dos verbos en el siglo XIII; estas variantes se introducen de forma tímida en el XIV y, en cambio, en el siglo XV solo resiste sin velarizar la $3{ }^{a}$ persona. En el segundo tiempo, el imperfecto de subjuntivo, los caminos de dir y prendre vuelven a coincidir en gran medida: desde un primer momento encontramos formas velarizadas de ambos verbos $\mathrm{y}$, en el siglo $\mathrm{xv}$, las formas innovadoras se convierten en mayoritarias. Por lo tanto, podríamos hablar de paralelismo entre estas dos subclases por lo que al tema de perfecto se refiere. La frecuencia de uso podría ayudarnos a entender el estadio de expansión en el que se encuentra la velar en cada uno de los tiempos. De este modo, en el tiempo más utilizado de este tema, es decir, el pretérito perfecto simple, vemos que las formas velarizadas aún son minoría, en parte porque la $3 .{ }^{a}$ persona, que es la más utilizada con diferencia, se muestra reticente al cambio. En cambio, en el imperfecto de subjuntivo, bastante menos usado, vemos cómo la velar penetra antes, y se expande mucho más rápidamente. Esto nos hace pensar que, a mayor frecuencia de uso, mayor reticencia a las formas analógicas velarizadas.

La comparativa entre los temas de presente de deure y prendre nos deja resultados diferentes a la extensión del afijo velar en los tiempos del patrón PyTA. Así, en el presente de indicativo, sí que vemos que la velar se introduce en el XIV y pasa a ser mayoritaria para ambos verbos en el xv, siguiendo el modelo de extensión a toda la clase del patrón PyTA. Sin embargo, en el presente de subjuntivo, la evolución de deure y prendre es dispar: en deure, como en el presente de indicativo, la velar se introduce en el xIV, pero desaparece de la documentación en el xv. Esta inversión de la tendencia podría deberse a una limitación de nuestro corpus, ya que los datos del presente de subjuntivo del CICA indican que la tendencia general es la expansión de la velar. Pero existen causas relacionadas con la estructura del paradigma que explican la reticencia de deure a generalizar la forma velar en la primera persona del presente de indicativo: como este verbo tenía una velar etimológica en el tema de perfecto, este afijo no se pudo generalizar en el tema de presente hasta que las formas 1 dec 'debí' y 3 dec 'debió' pasaron de fuertes a débiles: degui y degué, respectivamente, ya que, si no, se hubiera producido un sincretismo entre las formas del perfecto dec y la primera persona del presente de indicativo: deig > dec 'debo'. Por otra parte, también debió influir en el mantenimiento de la forma deig, con palatal final, la presencia de esta terminación en palatal en otros verbos de uso frecuente como 1 vaig 'voy'. Como la 1 . $^{a}$ persona del presente de indicativo se asocia regularmente con el presente de subjuntivo (patrón L), la conservación de deig debió favorecer el mantenimiento de las formas con palatal en toda la serie.

En cuanto al presente de subjuntivo de prendre, ya desde en el XIII aparecen formas velarizadas que, además, son mayoritarias desde este mismo siglo. El hecho de que el afijo velar en prendre no sea etimológico ni en el tema de presente ni en el tema de perfecto nos llevaría a esperar, en principio, que la velarización debería ser más tardía. Sin embargo, los datos nos indican todo lo contrario: en el tema de presente de prendre las formas innovadoras se extienden rápidamente, incluso más que en el verbo deure. Pueden ayudar a entender la rápida velarización de prendre varios factores: por una parte, la inexistencia en el paradigma de formas concurrentes de perfecto con una consonante velar, como ocurre con dec 'debí/debió', que frena la 
extensión de dec 'debo'; por otro, la posibilidad de evitar la homofonía entre las personas $1 .^{a}$ y $3 .^{a}$ del presente de indicativo, que originariamente tenían la forma pren, y que se diferencian con la adición de la consonante velar a la 1. ${ }^{a}$ persona: prenc.

Como conclusión global, hemos visto que la expansión de la velar en el tiempo no es un proceso homogéneo, ni en las formas del patrón L, ni en las del patrón PyTA. En el primero, hemos visto que, dependiendo de verbos y de su idiosincrasia particular, la velar se introduce antes y se propaga rápidamente (prendre), o tarda más en introducirse y su extensión es más lenta (deure). Por lo que respecta a las formas del segundo patrón, hemos visto que en los dos verbos que hemos analizado (dir y prendre) la evolución es paralela. Así, en el pretérito perfecto simple la introducción es más tardía y la propagación más lenta, mientras que en el imperfecto de subjuntivo las formas innovadoras aparecen ya en el siglo XIII y se extienden muy rápidamente. Las formas velarizadas acaban generalizándose en el conjunto del patrón L y PyTA, pero, contrariamente a lo que se podría esperar, no es un proceso homogéneo dentro de las formas de cada patrón ni en el conjunto de verbos afectados. 


\section{BIBLIOGRAFÍA}

Coromines, J. (1971): Lleures i converses d'un filòleg, Barcelona: Club Editor.

Gould, S.J. y E.S. VRba (1982): «Exaptation-a Missing Term in the Science of Form», Paleobiology 8(1): 4-15.

Harris, A.C. y L. Campbell (1995): Historical syntax in cross-linguistic perspective, Cambridge: Cambridge University Press.

Lass, R. (1990): «How to Do Things with Junk: Exaptation in Language Evolution», Journal of Linguistics 26(1): 79-102.

Maiden, M. (2018): The romance verb: morphomic structure and diachrony, Oxford: Oxford University Press.

Mascaró, J. (1983): La fonologia catalana i el cicle fonològic, Bellaterra: Servei de Publicacions de la Universitat Autònoma de Barcelona.

Mascaró, J. (1985): Morfologia, Barcelona: Enciclopèdia Catalana.

Meyer-Lüвкe, W. (1895): Grammaire des langues romanes, vol. 2: Morphologie, París: Welter.

Moll, F. de B. (2006): Gramàtica històrica catalana (J. Martí Mestre y J. Jiménez Martínez, eds.), València: Universitat de València.

O’Neill, P. (2015): «The Origin and Spread of Velar Allomorphy in the Spanish Verb: A Morphomic Approach», Bulletin of Hispanic Studies 92: 489-518.

PÉREz SALDANYA, M. (1996): «Analogia i canvi morfològic: a propòsit de les formes verbals velaritzades», Caplletra 19: 279-305.

Pérez Saldanya, M. (1998): Del llatí al català: morfosintaxi verbal històrica, València: Universitat de València.

PÉrez Saldanya, M. (2013): “Que sa il-lustre senyoria cullga o faça cullir dits fruits”: una aproximació històrica als verbs velaritzats», en E. Clua y M.R. Lloret (eds.), Qüestions de morfologia flexiva i lèxica del català. Volum d'homenatge a Joaquim Viaplana, Alacant: Institut Interuniversitari de Filologia Valenciana, 313-333.

Ronjat, J. (1937): Grammaire istorique des parlers provençaux modernes, vol. 3, Montpellier: Société des Langues Romanes.

Querol, L. (2011): La morfologia verbal del català nord-occidental: descripció i anàlisi dels segments velar i palatal, Lleida: Pagès.

Viaplana, J. (1986): «Morfologia flexiva i flexió verbal catalana», Llengua i Literatura 1: 385-403.

Wall, A. y Á.S. Octavio de Toledo y Huerta (2016): «Exploring and recycling», en M. Norde y F. Van de Velde (eds.), Exaptation and Language Change, Amsterdam: John Benjamins Publishing Company, 341-375.

WheELer, M.W. (2007): Morfologia i fonologia catalana i romànica: estudis diacrònics, València / Barcelona: Institut Interuniversitari de Filologia Valenciana / Publicacions de l'Abadia de Montserrat.

Wheeler, M.W. (2011): «The Evolution of a Morphome in Catalan Verb Inflection», en M. Maiden, J.C. Smith, M. Goldbach y M.O. Hinzelin (eds.), Morphological Autonomy: Perspectives From Romance Inflectional Morphology, Oxford: Oxford University Press, 182-209. 
